\title{
The Neural Basis of Interindividual Variability in Inhibitory Efficiency after Sleep Deprivation
}

\author{
Y. M. Lisa Chuah, ${ }^{1}$ Vinod Venkatraman, ${ }^{1}$ David F. Dinges, ${ }^{2}$ and Michael W. L. Chee ${ }^{1}$ \\ ${ }^{1}$ Cognitive Neuroscience Laboratory, Singapore Health Services, Singapore, 169611, and ${ }^{2}$ Department of Psychiatry, University of Pennsylvania School of \\ Medicine, Philadelphia, Pennsylvania 19104-6021
}

\begin{abstract}
Sleep deprivation results in the loss of our ability to suppress a prepotent response. The extent of decline in this executive function varies across individuals. Here, we used functional magnetic resonance imaging to study the neural correlates of sleep deprivation-induced differences in inhibitory efficiency. Participants performed a go/no-go task after normal sleep and after $24 \mathrm{~h}$ of total sleep deprivation. Regardless of the extent of change in inhibitory efficiency, sleep deprivation lowered go/no-go sustained, task-related activation of the ventral and anterior prefrontal (PFC) regions bilaterally. However, individuals better able to maintain inhibitory efficiency after sleep deprivation could be distinguished by lower stop-related, phasic activation of the right ventral PFC during rested wakefulness. These persons also showed a larger rise in such activation both here and in the right insula after sleep deprivation relative to those whose inhibitory efficiency declined.
\end{abstract}

Key words: go/no-go; inhibition; error monitoring; compensation; functional neuroimaging; prefrontal cortex

\section{Introduction}

A range of executive functions that rely on inhibition are adversely affected by sleep deprivation. This results in cognitive inflexibility, impaired decision making (Harrison and Horne, 1999, 2000; Nilsson et al., 2005), deficient error detection (Nilsson et al., 2005; Tsai et al., 2005), and impairment of various aspects of executive attention (Jennings et al., 2003; Drummond et al., 2005b; Durmer and Dinges, 2005). Although everyone will experience such cognitive deficits if such deprivation continues unabated for days, less severe loss of sleep (up to $40 \mathrm{~h}$ awake) results in surprisingly large differences between subjects in the magnitude of cognitive deficits. Among healthy sleep-deprived adults, the distribution of responses to sleep loss ranges from apparent cognitive resistance to severe cognitive impairment (Doran et al., 2001). Importantly, such differential neurocognitive vulnerability to sleep deprivation shows evidence of a stable trait (Van Dongen et al., 2004, 2005; Van Dongen, 2005a), suggesting that it has a reliable neural basis. Preliminary functional brain imaging studies have also reported correlations between brain activation and performance decline after sleep deprivation (Caldwell et al., 2005; Mu et al., 2005; Chee et al., 2006).

We hypothesized that deficient recruitment of brain regions involved in inhibitory processes would be observed in individuals who are vulnerable to sleep deprivation. To investigate this possibility, we used a variant of the go/no-go task (see Fig. 1) (Gara-

\footnotetext{
Received March 1, 2006; revised May 26, 2006; accepted May 26, 2006.

This work was supported by DSO National Laboratories, Singapore Grant DSOCL05141. We thank Julian Lim and Dr. Wei-Chieh Choo for the recruitment of participants and assistance in the collection of data.

Correspondence should be addressed to Dr. Michael W. L. Chee, Cognitive Neuroscience Laboratory, Singapore Health Services, 7 Hospital Drive, \#01-11, Block B, SingHealth Research Facilities, Singapore, 169611. E-mail: mchee@pacific.net.sg.

DOI:10.1523/JNEUROSCI.0906-06.2006

Copyright $\odot 2006$ Society for Neuroscience $\quad 0270-6474 / 06 / 267156-07 \$ 15.00 / 0$
}

van et al., 2002, 2003) that requires inhibition of the irrelevant response as well as ongoing error monitoring (Konishi et al., 1998; Garavan et al., 1999; Hester et al., 2004). Successful response inhibition (stops) has been shown to activate the right inferior lateral prefrontal cortex (PFC) (Konishi et al., 1998; Garavan et al., 1999), whereas errors of commission (errors) have been associated with activation of the anterior cingulate cortex and the medial frontal gyrus (Garavan et al., 2003; Rubia et al., 2003; Hester et al., 2004, 2005). These regions are considered to be crucial for the higher-order, cognitive control of behavior, with the anterior cingulate being important for conflict monitoring (Carter et al., 1998; Braver et al., 2001) and the inferior prefrontal cortex for sustained attentional control (Braver et al., 2003; Egner and Hirsch, 2005) as well as the suppression of irrelevant responses (Aron et al., 2004). The modulation of activation in these regions as a result of $24 \mathrm{~h}$ of sleep deprivation was the focus of the present study.

\section{Materials and Methods}

Participants. Participants were selected from respondents to a web-based questionnaire. They had to (1) be right-handed, (2) be between 18 and 35 years of age, (3) have habitual good sleeping habits (sleeping no less than $6.5 \mathrm{~h}$ each night for the past 1 month), (4) score no more than 22 on the Morningness-Eveningness scale (Horne and Ostberg, 1976), (5) not be on any long-term medications, (6) have no symptoms associated with sleep disorders, and (7) have no history of any psychiatric or neurologic disorders. The sleeping habits of all participants were monitored throughout the 2 week duration of the study, and only those whose actigraphy data indicated habitual good sleep (i.e., they usually slept no later than 1:00 A.M. and woke no later than 9:00 A.M.) were recruited for the study after informed consent.

Twenty-seven persons successfully completed this study. They were right-handed, healthy, university undergraduates and graduates (12 females; mean \pm SD age, $21.5 \pm 1.70$ years; range, $19-26$ years). All par- 

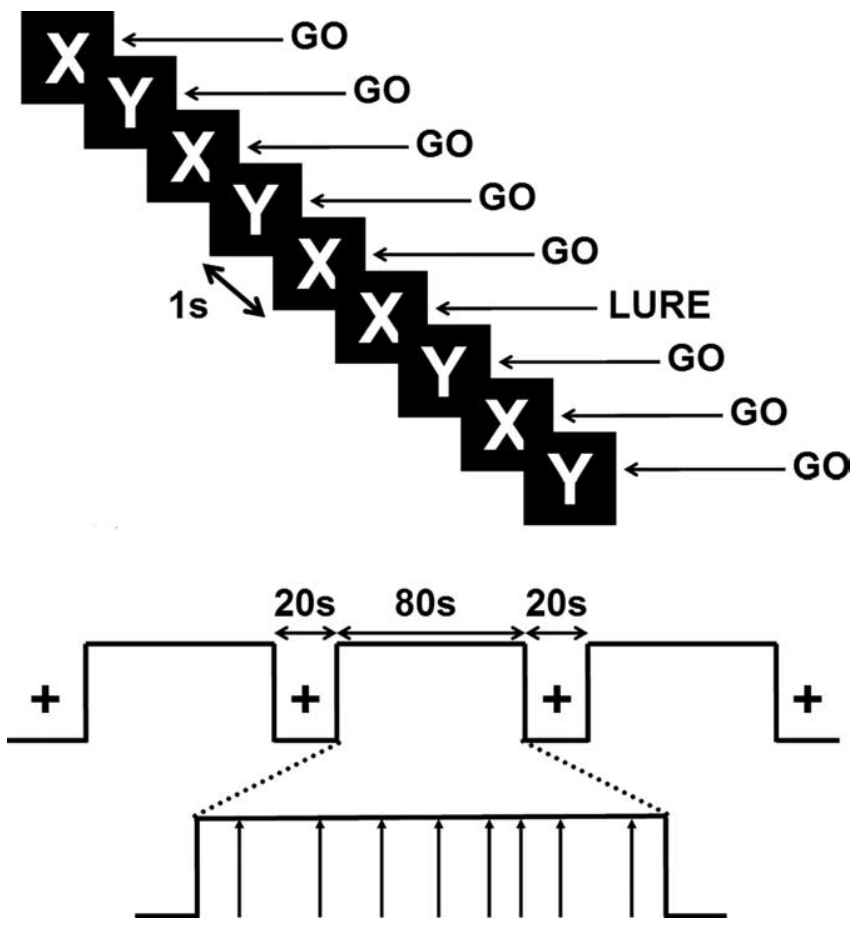

Figure 1. Schematic representation of the go/no-go task and the timing parameters of a single run. Participants performed four runs of the task in the scanner during each scanning session. A mixed design incorporating block and event-related features was used. Blocks of fixation were interspersed with task blocks. Within each task block, there were eight lure trials (as represented by the arrows) at random intervals of $4,6,8$, and $10 \mathrm{~s}$. Activation for the blocks represent tonic, sustained activity associated with task performance, whereas event-related activation represented the transient activity associated with stops and errors.

ticipants indicated that they did not smoke or consume any medications, stimulants, caffeine, or alcohol for at least $24 \mathrm{~h}$ before scanning.

Experimental task. The go/no-go task was based on previous work by Garavan and colleagues (Garavan et al., 2002, 2003; Hester et al., 2004). The letters $X$ and $Y$ were presented in a serial, alternating pattern at $1 \mathrm{~Hz}$. Participants were instructed to make a button press to every letter except when the alternation was interrupted (i.e., they were to withhold the response when there is a repetition of a letter). To ensure a comparable number of stops and errors, the stimulus duration for each participant was determined during prescanning testing (see below).

A mixed design, incorporating block and event-related features, was used (Visscher et al., 2003; Donaldson, 2004). Each run consisted of four fixation blocks interleaved with three task blocks (Fig. 1). The first fixation block lasted $28 \mathrm{~s}$ (data for the first $8 \mathrm{~s}$ was discarded to allow for steady-state magnetization), whereas the remaining three fixation blocks lasted 20 s. Each task block consisted of 80 trials, of which 72 were go trials and eight were lure trials (i.e., a response was to be withheld). The inter-lure intervals were randomly distributed between $4,6,8$, and $10 \mathrm{~s}$ (with an average of $7.5 \mathrm{~s}$ ).

Study procedure. All participants visited the laboratory three times. They first attended a briefing session during which they were informed of the protocol and requirements of the study and were given extensive practice on the go/no-go task. Each participant completed eight runs of the go/no-go task in which there was a decrement of $100 \mathrm{~ms}$ in stimulus presentation duration with each following run. In the first run, each stimulus was presented for $900 \mathrm{~ms}$, followed by an interstimulus interval (ISI) of $100 \mathrm{~ms}$ fixation. In the second run, stimulus duration was $800 \mathrm{~ms}$ with an ISI of $200 \mathrm{~ms}$ fixation, and, in the final practice run, stimulus duration was $200 \mathrm{~ms}$ with an ISI of $800 \mathrm{~ms}$ fixation. This process ensured that participants were well practiced and additionally generated individualized timing parameters that would subsequently elicit an approximately equivalent number of stops and errors in the in-scanner experiment (Garavan et al., 2002).
The first scanning session took place $\sim 1$ week later. The order of the two sessions (rested wakefulness and sleep deprivation) was counterbalanced across all of the participants to minimize possible effects of practice on brain activation (Van Dongen, 2005b). The 1 week interval between scan sessions (mean $\pm \mathrm{SD}, 7.78 \pm 2.36 \mathrm{~d}$ ) sought to minimize the possibility of residual effects of sleep deprivation on cognition for those participants whose sleep deprivation session had preceded their rested wakefulness session (Van Dongen et al., 2003).

The scans at rested wakefulness took place at 8:00 A.M. For the sleep deprivation session, participants were monitored in the laboratory from 6:30 P.M. onward, and scanning took place at 7:00 A.M. the next morning. At the beginning of the sleep deprivation session, participants completed the Raven's Advanced Progressive Matrices (Raven et al., 1998), the Barrett Impulsiveness Scale-11 (Patton et al., 1995), the Cognitive Failures Questionnaire (Broadbent et al., 1982), and the Sixteen Personality Factor Scale (Cattell et al., 2002). Hereafter, at every hour from 8:00 P.M. to 5:00 A.M., they completed $10 \mathrm{~min}$ of the Psychomotor Vigilance Task (Dinges et al., 1997), the Karolinska Sleepiness Scale (Akerstedt and Gillberg, 1990), and a Likert-type rating scale (0-10) of motivation, fatigue, and mood, which were anchored by the terms motivated-unmotivated, fresh-exhausted, elated-depressed, congenial-irritable, relaxed-stressed, and calm-anxious (henceforth referred to as the mood scale). For the remaining time, they were allowed to engage in nonstrenuous activities such as reading and conversing.

Immediately before entering the scanner, participants completed 10 min of the Psychomotor Vigilance Task, the mood scale, and a practice run of the go/no-go task. Ratings on the Karolinska Sleepiness Scale were obtained after the practice run. Every participant was then scanned on four runs of the task. Performance on the scanner task was continuously monitored, and participants were prompted to respond through the intercom system whenever they failed to respond to three consecutive go trials. There was an average of 0.30 prompts given during the rested wakefulness session, and the average number of prompts for the sleep deprivation session was 1.33 .

Ratings on the Karolinska Sleepiness Scale were also obtained (using the button box) at the end of each in-scanner run. Self-perceived sleepiness at each state was obtained by averaging the five ratings of sleepiness given on this scale after the five runs of the go/no-go task (one practice run and four in-scanner test runs). Ratings of motivation and mood were similarly derived by averaging the responses before and after each scanning session for each item on the mood scale. The number of lapses on the trial performed just before each scanning session was treated as the index of psychomotor vigilance (Dinges et al., 1997).

Imaging procedure and analysis. Stimuli were projected onto a screen using a liquid crystal display projector and viewed by participants through a rearview mirror. Participants responded using a button box held in the right hand. A bite bar and foam padding were used to reduce head motion. Images were acquired on a 3T Allegra system (Siemens, Erlangen, Germany). A gradient echo-planar imaging sequence was used with a repetition time of $2000 \mathrm{~ms}$, field of view of $192 \times 192 \mathrm{~mm}$, and a $64 \times 64 \mathrm{~mm}$ pixel matrix. Thirty-two oblique axial slices $(3 \mathrm{~mm}$ thick with a $0.3 \mathrm{~mm}$ interslice gap), approximately parallel to the anterior commissure-posterior commissure line, were acquired. High-resolution coplanar T1 anatomical images were also obtained. For the purpose of image display on Talairach space, an additional high-resolution anatomical reference image was acquired using a three-dimensional magnetization-prepared rapid-acquisition gradient echo sequence.

The functional images were processed using Brain Voyager QX version 1.5.2 (Brain Innovation, Maastricht, The Netherlands). Intra-session image alignment to correct for motion across runs was performed using, as the reference image, the first image of the functional run that was acquired immediately before the coplanar T1-weighted image. Interslice timing differences attributable to slice acquisition order were adjusted using linear interpolation. Gaussian filtering was applied in the spatial domain using a smoothing kernel of $4 \mathrm{~mm}$ full-width at half-maximum (FWHM) for individual activation maps and at $8 \mathrm{~mm}$ FWHM for group level activation maps. After linear trend removal, a high-pass filter of $160 \mathrm{~s}$ was applied. The T1 images were used to register the functional dataset to the volunteers' own three-dimensional image, and the result- 
ing aligned dataset was transformed into Talairach space. The group-level anatomical image was an arithmetical average of the volunteers' structural images.

The functional image data were analyzed for both sessions using a general linear model with one block predictor (task) and two eventrelated predictors (stops, errors). Because there was an increased tendency for omissions after sleep deprivation (Table 1), only stop trials that were not preceded or followed by an omission were modeled. This was to increase the likelihood that the lure trials without responses were stops as opposed to errors of omission. All predictors were convolved using a canonical hemodynamic response function and analyzed using a mixed-effects model. In this model, modulation of blood oxygenation leveldependent signal at the time of task blocks, relative to signal recorded during periods of fixation, was taken to represent tonic or sustained activation associated with sustained attention required to respond to the go/no-go task. In these task blocks, go trials comprised $90 \%$ of the stimuli. Transient or phasic signal changes elicited by successful inhibition associated with stops or errors were modeled as discrete events occurring within the task blocks.

Data analysis. Inhibitory efficiency was indexed using the intra-individual variability in reaction time on the go trials [intra-individual coefficient of variation (ICV)] (West et al., 2002; Stuss et al., 2003; Castellanos et al., 2005). The appropriateness of ICV as an index of inhibitory efficiency was corroborated by the correlations between ICV during rested wakefulness and other subjective measures of impulsiveness and self-control (supplemental Table 1, available at www.jneurosci.org as supplemental material).

To characterize the effects of sleep deprivation on brain activation, direct contrasts were obtained across the two sessions for eventrelated changes associated with stops and errors as well as the sustained task-related activation (covering both go and lure trials). Unless otherwise specified, a threshold of $p<.001$ (uncorrected) was used in the analysis of contrasts in this study.

Vulnerability to sleep deprivation was computed on the basis of the extent of an individual's change in inhibitory efficiency on the go/no-go task after sleep deprivation, taking into account their performance at rested wakefulness, using the following equation: $\left(\mathrm{ICV}_{\mathrm{SD}}-\mathrm{ICV}_{\mathrm{RW}}\right) \times \mathrm{ICV}_{\mathrm{RW}}$, where $\mathrm{SD}$ is sleep deprivation and RW is rested wakefulness. Participants were divided into tertiles, based on the extent of their change in performance, to facilitate visualization and discussion of the relationships between activation and vulnerability to sleep deprivation. These three groups, comprising nine subjects each, were termed low vulnerable, moderately vulnerable, and highly vulnerable. Because the focus was on the relationship between inhibitory efficiency and interindividual variability in vulnerability to sleep loss and evaluating whether activation at rested wakefulness can help predict vulnerability to sleep loss, parameter estimates were obtained from functionally defined regions-of-interest (ROIs) that were significantly activated at rested wakefulness for stops and errors (for ROI, see Table 2). Using such a functional ROI approach helps to constrain hypothesis testing concerning state effects to regions known to be robustly activated by the cognitive process of interest. The voxels contributing to each ROI lay within a bounding cube of edge $10 \mathrm{~mm}$ surrounding the peak activation for that ROI. These parameter estimates were then analyzed separately using a 2 (state) by 3 (group) repeated-measures ANOVA. All analyses involving behavioral variables and parameter estimates were conducted using SPSS version 13 , and significance was determined using an $\alpha$ level of 0.05 .

\section{Results}

\section{Behavioral findings}

The data for 27 participants was analyzed, but, because of a technical error, a participant's behavioral ratings of motivation and mood during the sleep deprivation session were not recorded. Variability in go/no-go performance increased significantly after sleep deprivation, along with significant decrements in hit rate and stop rate (Table 1). Accompanying this decline in inhibitory efficiency were increased lapses in psychomotor vigilance, a greater subjective sense of sleepiness, as well as decrements in ratings of motivation and mood (supplemental Table 2, available at www.jneurosci.org as supplemental material).

The three groups differentiated by extent of change in their 

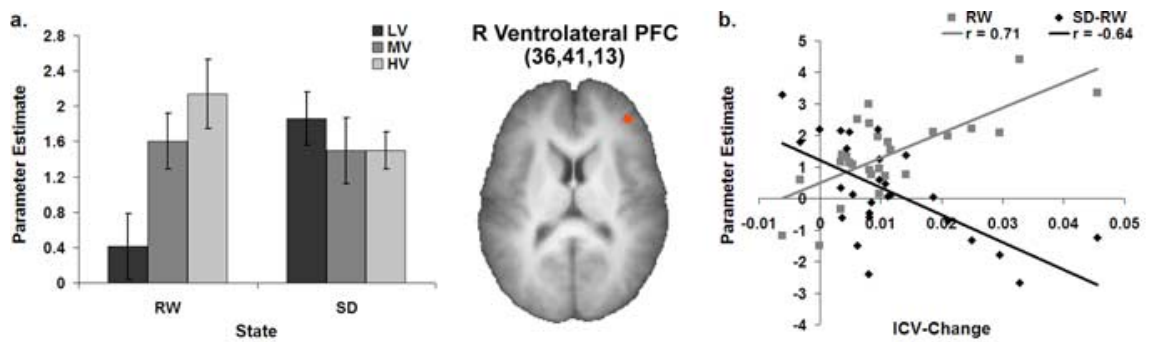

Figure 2. The parameter estimates ( \pm SEM) of activation in the right $(\mathrm{R})$ ventrolateral PFC for stops are plotted as a function of state (RW, rested wakefulness; SD, sleep deprivation) and group (LV, low vulnerability; MV, moderate vulnerability; HV, high vulnerability; $n=9$ in each group) (a). There was a significant interaction of state by group: individuals who best maintained inhibitory efficiency after sleep deprivation (LV) had the lowest activation at rested wakefulness, whereas those most vulnerable to sleep loss (HV) showed the highest activation. After sleep deprivation, this pattern was reversed. As such, the change in activation in this region after sleep deprivation (relative to RW) as well as the level of activation at rested wakefulness were negatively correlated with sleep deprivation-related decrease in inhibitory efficiency $(\boldsymbol{b})$.
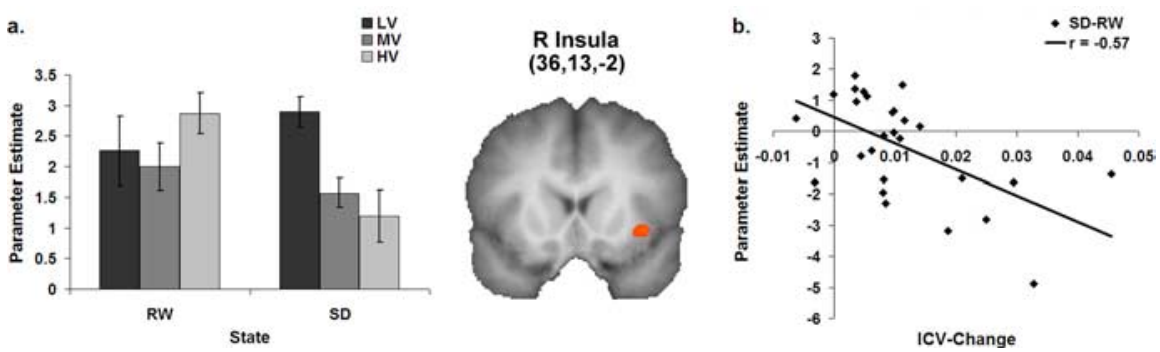

Figure 3. The average \pm SEM parameter estimates of activation in the right $(R)$ insula for stops are plotted as a function of state (RW, rested wakefulness; SD, sleep deprivation) and group (LV, low vulnerability; MV, moderate vulnerability; HV, high vulnerability; $n=9$ in each group) (a). There was a significant interaction of state by group, reflecting an increase in activation for those least vulnerable to sleep deprivation (LV), whereas activation decreased in the other groups, with the greatest decrease seen for the HV group. There was also a significant correlation between the sleep deprivation-related change in activation and the change in inhibitory efficiency $(\boldsymbol{b})$.

inhibitory efficiency after sleep deprivation $\left(F_{(2,24)}=8.97 ; p=\right.$ 0.001 ) (supplemental Table 3, available at www.jneurosci.org as supplemental material) but were otherwise comparable on other behavioral measures. Post hoc analyses (Scheffé's) indicated that the highly vulnerable group differed significantly from both the low-vulnerable $(p=0.002)$ and moderately vulnerable ( $p=$ 0.02 ) groups in their inhibitory efficiency after sleep deprivation, whereas the difference between the low-vulnerable and moderately vulnerable groups was not significant $(p=0.61)$. Repeatedmeasures ANOVA conducted on the other behavioral measures (self ratings of sleepiness, motivation, mood, and psychomotor vigilance) (supplemental Table 3, available at www.jneurosci.org as supplemental material) indicated that the groups did not differ in their manner of change after sleep deprivation on these other measures. There were also no differences between the groups in terms of age, stimulus presentation rate on the go/ no-go task, nonverbal intelligence quotient score, and reported impulsiveness (supplemental Table 4, available at www.jneurosci. org as supplemental material).

\section{Activation during rested wakefulness}

As expected, there was a right-hemisphere dominance in activation for stops and errors (Table 2) (Garavan et al., 1999, 2002, 2003; Hester et al., 2004; Kelly et al., 2004). With stops, activation was seen in the right inferior frontal gyrus/ventrolateral PFC, right middle frontal gyrus, right inferior parietal lobule, and right lenticular nucleus, whereas activation in the insula was bilateral. Areas significantly activated by errors included the right anterior cingulate, right medial frontal gyrus, right inferior parietal lob- ule, and the bilateral insula regions (for regions showing significant deactivation, see supplemental Table 5, available at www.jneurosci.org as supplemental material).

Tonic task-related activation was seen bilaterally in the medial frontal gyrus, precentral regions, superior parietal lobules, and caudate, whereas activation for the insula was confined to the right hemisphere. Task-related deactivation was seen in the left precuneus/posterior cingulate that extended bilaterally and also in the bilateral postcentral gyrus.

\section{Effects of sleep deprivation on event- related activation in the ventrolateral PFC and right insula}

Considering the entire cohort, there were no significant changes as a result of sleep deprivation for both stops and errors. However, underscoring the importance of considering interindividual variability in response to sleep loss, a different picture emerged when group differences in sleep loss vulnerability were considered. In the latter analyses, there were significant interactions of state and group in the right ventrolateral PFC $\left(F_{(2,24)}=6.33 ; p=0.006\right)$ and right insula $\left(F_{(2,24)}=6.15 ; p=0.007\right)$ for stops (see Figs. 2, 3). A significant effect of group was present in the right anterior cingulate $\left(F_{(2,24)}=4.54 ; p=0.02\right)$ for errors (see Fig. 4). The findings in each of these three regions are now reported in greater detail.

At rested wakefulness, there were significant differences between the groups for activation in the right ventrolateral PFC $\left(F_{(2,24)}=5.93 ; p=0.008\right)$. Individuals who best maintained inhibitory efficiency after sleep deprivation showed lower activation at rested wakefulness in the right ventrolateral PFC compared with vulnerable individuals (Fig. 2). Importantly, resistant individuals appeared able to transiently increase right ventrolateral PFC activation after sleep deprivation $\left(F_{(1,8)}=12.29 ; p=\right.$ $0.008)$ whereas the highly vulnerable group $\operatorname{did} \operatorname{not}\left(F_{(1,8)}=2.31\right.$; $p=0.17$ ).

These results held up in the correlation analyses. Greater activation of the ventrolateral PFC at rested wakefulness correlated highly with the decline in inhibitory efficiency after sleep deprivation $(r=0.71 ; p<0.001)$, whereas greater relative increase in activation after sleep deprivation correlated with less decline in inhibitory efficiency $(r=-0.64 ; p<0.001)$ (Fig. 2).

At rested wakefulness, all three groups activated the right insula similarly $\left(F_{(2,24)}=1.03 ; p=0.37\right)$ (Fig. 3). After sleep loss, whereas activation increased for those least vulnerable to sleep deprivation $\left(F_{(1,8)}=2.87 ; p=0.13\right)$, the other two groups evinced decreases in activation, with the greatest decrease seen for the highly vulnerable group $\left(F_{(1,8)}=8.57 ; p=0.02\right)$. Level of activation in the right insula for stops differed significantly between the groups after sleep deprivation $\left(F_{(2,24)}=7.92 ; p=\right.$ $0.002)$, as did the extent of sleep deprivation-related activation change $\left(F_{(2,24)}=6.15 ; p=0.007\right)$. Similar to the right ventrolateral PFC, the change in activation after sleep deprivation correlated with the extent of change in inhibitory efficiency $(r=$ 
$-0.57 ; p=0.002)$. However, activation at rested wakefulness in the insula did not correlate with behavioral performance change $(r=-0.21 ; p=0.30)$.

The effect of group found in the anterior cingulate was attributable to higher activation for the low-vulnerable group at rested wakefulness $\left(F_{(2,24)}=3.22 ; p=\right.$ $0.06)$. The groups did not differ in their activation when sleep deprived $\left(F_{(2,24)}=\right.$ 2.65; $p=0.09)$ and in their extent of change in activation after sleep deprivation $\left(F_{(2,24)}=0.38 ; p=0.69\right)$ (Fig. 4). There was a modest, although not significant, correlation between activation at rested wakefulness and change in inhibitory efficiency after sleep deprivation $(r=-0.30 ; p=0.13)$.

\section{Effects of sleep deprivation on sustained task-related activation}

There were significant effects of state in bilateral ventrolateral PFC (right $>$ left) and right anterior insula (Fig. 5). The decrease in activation in these regions after sleep deprivation was observed across all levels of vulnerability to sleep deprivation, with there being no significant effect of group or significant interaction of state and group for any region ( smallest $p$ value $=0.35$ ).

\section{Discussion}

Twenty-four hours of sleep deprivation resulted in decline of sustained task-related activation in ventral and anterior prefrontal regions in all subjects regardless of their vulnerability to sleep deprivation. However, transient, phasic activation of the right ventrolateral PFC and right insula in response to successful inhibitions (stops) and the anterior cingulate for errors varied depending on the degree to which an individual's inhibitory efficiency was affected after sleep loss.

The ventrolateral PFC, insula, and anterior cingulate are regions frequently implicated in studies of inhibition and cognitive control (Wager et al., 2005). Different subregions within the right ventrolateral PFC were associated with processing stops and maintaining go responses. We postulate that the event-related activation seen in the right ventrolateral PFC for stops is related to the suppression of the prepotent but inappropriate response (Sylvester et al., 2003; Aron et al., 2004), whereas the bilateral tonic task-related activation located more anteriorly is associated with maintaining sustained cognitive control (Braver et al., 2003; Egner and Hirsch, 2005) and/or sustained attention (Yamasaki et al., 2002; Lawrence et al., 2003; Drummond et al., 2005a).

The ability to maintain inhibitory efficiency in the go/no-go task after sleep loss appears to be related to the transient activity in the right ventrolateral PFC and insula for stops. It is important to note that inhibitory efficiency did not differ significantly between the three groups at rested wakefulness, although there was a trend toward a higher variability in go/no-go performance for the group most vulnerable to the effects of sleep loss. This suggests that implementing inhibition for stops may already be comparatively difficult for vulnerable individuals at rested wakefulness, and the higher right ventrolateral PFC may have been necessary for satisfactory performance on the go/no-go task. Higher activation in this region after normal sleep has been associated with poorer inhibitory performance (Bellgrove et al., 2004; Wager et al., 2005).

After sleep deprivation, vulnerable individuals appear to have
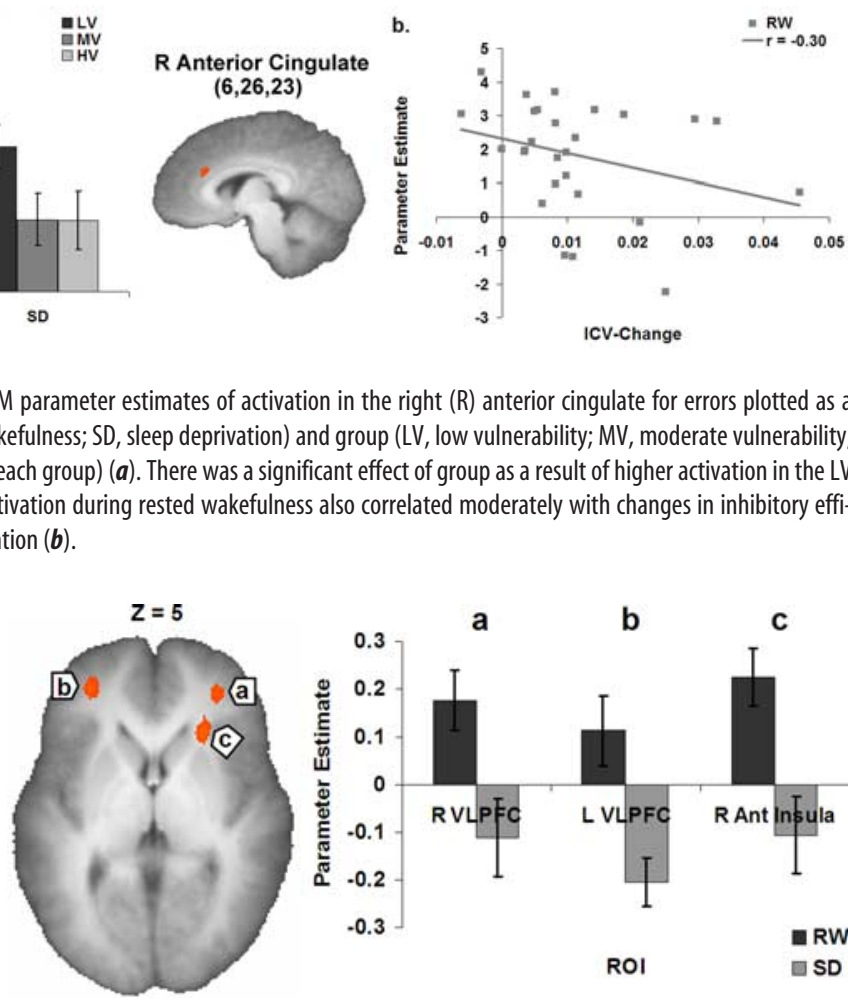

Figure 5. Parameter estimates of activation in regions associated with tonic, task-related activation that show a significant effect of state. The regions are the right ventrolateral PFC ( 30 , $41,7)(\boldsymbol{a} ;$ RVLPFC), the left ventrolateral PFC $(-30,44,7)(\boldsymbol{b}$; LVLPFC), and the right anterior insula $(24,25,4)$ ( $\boldsymbol{c}$; R Ant Insula). RW, Rested wakefulness; SD, sleep deprivation.

difficulty recruiting the ventrolateral PFC, whereas resistant individuals are able to do so. The present finding is reminiscent of the situation in mild cognitive impairment (MCI), in which increased hippocampal activation in these individuals is thought to enable them to engage in associative encoding to a level comparable with control subjects (Dickerson et al., 2005). In that study, increased hippocampal activation in MCI subjects preceded the precipitous decline in both activation and memory performance observed in demented volunteers.

We posit that the significant increase in right ventrolateral PFC phasic activation in the less vulnerable individuals after sleep deprivation represents a compensatory response to decreases in the tonic task-related activation in the bilateral anterior ventrolateral PFC and right anterior insula.

The anterior cingulate is known to work in concert with the lateral prefrontal cortex in the implementation of cognitive control, through the detection of response conflict (Carter et al., 1998; MacDonald et al., 2000) and/or in the general monitoring for errors (Garavan et al., 2003; Hester et al., 2004). The more efficient (lower activation) recruitment of the right ventrolateral PFC for stops at rested wakefulness in those less vulnerable to sleep deprivation may be related to higher activation of the anterior cingulate for errors in this group. There was a negative, although nonsignificant, correlation between activation in the ventrolateral PFC and anterior cingulate at rested wakefulness.

The role of the insula in inhibition remains to be clarified, although its activation has been reported frequently in functional magnetic resonance imaging studies of the go/no-go task (Garavan et al., 1999; Kelly et al., 2004). This region has been implicated in motivation, affect, pain, and emotional processing (Ploghaus et al., 1999; Damasio et al., 2000; Dolan, 2002; Phan et al., 2004; 
Wang et al., 2005). The differential change in activation between the groups (increase after sleep deprivation for those less vulnerable and a decrease for those most vulnerable) suggests a compensatory role for the insula in the context of sleep deprivation and is consistent with the posited "insular-prefrontal-cingulate network" underlying inhibition (Wager et al., 2005). Although a plausible manner in which activation in this region could be relevant to the go/no-go task lies in emotional/motivation differences between the groups, we found no significant group differences in self-ratings of motivation and affect.

Some researchers have argued that interindividual differences in the context of sleep deprivation are best seen in regions in which a relationship between activation and performance only emerges after sleep loss (Drummond et al., 2005a), akin to the pattern of activation change that was seen in the right insula for stops. The present results additionally illustrate the benefits of evaluating regions that show between-subjects differences in activation at rested wakefulness, such as the right ventrolateral PFC.

The present results also highlight the importance of taking into account interindividual differences in response to sleep loss when assessing the effects of sleep deprivation on cognition. Finally, although sleep deprivation has been postulated to particularly affect the prefrontal cortex (Horne, 1988; Harrison et al., 2000; Jones and Harrison, 2001; Mazur et al., 2002), it is clear that a compensatory response to maintain inhibitory efficiency may also arise from the PFC, dependent on some extent to which this region is recruited to perform the task on a normal day. The further characterization of how sleep deprivation modulates engagement of brain regions critical to the maintenance of task performance could have economic and clinical significance (Van Dongen et al., 2005).

\section{References}

Akerstedt T, Gillberg M (1990) Subjective and objective sleepiness in the active individual. Int J Neurosci 52:29-37.

Aron AR, Robbins TW, Poldrack RA (2004) Inhibition and the right inferior frontal cortex. Trends Cogn Sci 8:170-177.

Bellgrove MA, Hester R, Garavan H (2004) The functional neuroanatomical correlates of response variability: evidence from a response inhibition task. Neuropsychologia 42:1910-1916.

Braver TS, Barch DM, Gray JR, Molfese DL, Snyder A (2001) Anterior cingulate cortex and response conflict: effects of frequency, inhibition and errors. Cereb Cortex 11:825-836.

Braver TS, Reynolds JR, Donaldson DI (2003) Neural mechanisms of transient and sustained cognitive control during task switching. Neuron 39:713-726.

Broadbent DE, Cooper PF, FitzGerald P, Parkes KR (1982) The Cognitive Failures Questionnaire (CFQ) and its correlates. Br J Clin Psychol 21:1-16.

Caldwell JA, Mu Q, Smith JK, Mishory A, Caldwell JL, Peters G, Brown DL, George MS (2005) Are individual differences in fatigue vulnerability related to baseline differences in cortical activation? Behav Neurosci 119:694-707.

Carter CS, Braver TS, Barch DM, Botvinick MM, Noll D, Cohen JD (1998) Anterior cingulate cortex, error detection, and the online monitoring of performance. Science 280:747-749.

Castellanos FX, Sonuga-Barke EJ, Scheres A, Di Martino A, Hyde C, Walters JR (2005) Varieties of attention-deficit/hyperactivity disorder-related intra-individual variability. Biol Psychiatry 57:1416-1423.

Cattell RB, Cattell AKS, Cattell HEP (2002) 16PF fifth edition administrator's manual. Champaign, IL: Institute for Personality and Ability Testing.

Chee MWL, Chuah YML, Venkatraman V, Chan WY, Philip P, Dinges DF (2006) Functional imaging of working memory following normal sleep and after 24 and 35 hours of sleep deprivation: correlations of frontoparietal activation with performance. NeuroImage 31:419-428.

Damasio AR, Grabowski TJ, Bechara A, Damasio H, Ponto LL, Parvizi J,
Hichwa RD (2000) Subcortical and cortical brain activity during the feeling of self-generated emotions. Nat Neurosci 3:1049-1056.

Dickerson BC, Salat DH, Greve DN, Chua EF, Rand-Giovannetti E, Rentz DM, Bertram L, Mullin K, Tanzi RE, Blacker D, Albert MS, Sperling RA (2005) Increased hippocampal activation in mild cognitive impairment compared to normal aging and AD. Neurology 65:404-411.

Dinges DF, Pack F, Williams K, Gillen KA, Powell JW, Ott GE, Aptowicz C, Pack AI (1997) Cumulative sleepiness, mood disturbance, and psychomotor vigilance performance decrements during a week of sleep restricted to $4-5$ hours per night. Sleep 20:267-277.

Dolan RJ (2002) Emotion, cognition, and behavior. Science 298:1191-1194. Donaldson DI (2004) Parsing brain activity with fMRI and mixed designs: what kind of a state is neuroimaging in? Trends Neurosci 27:442-444.

Doran SM, Van Dongen HP, Dinges DF (2001) Sustained attention performance during sleep deprivation: evidence of state instability. Arch Ital Biol 139:253-267.

Drummond SP, Meloy MJ, Yanagi MA, Orff HJ, Brown GG (2005a) Compensatory recruitment after sleep deprivation and the relationship with performance. Psychiatry Res 140:211-223.

Drummond SP, Bischoff-Grethe A, Dinges DF, Ayalon L, Mednick SC, Meloy MJ (2005b) The neural basis of the psychomotor vigilance task. Sleep 28:1059-1068.

Durmer JS, Dinges DF (2005) Neurocognitive consequences of sleep deprivation. Semin Neurol 25:117-129.

Egner T, Hirsch J (2005) Cognitive control mechanisms resolve conflict through cortical amplification of task-relevant information. Nat Neurosci 8:1784-1790.

Garavan H, Ross TJ, Stein EA (1999) Right hemispheric dominance of inhibitory control: an event-related functional MRI study. Proc Natl Acad Sci USA 96:8301-8306.

Garavan H, Ross TJ, Murphy K, Roche RA, Stein EA (2002) Dissociable executive functions in the dynamic control of behavior: inhibition, error detection, and correction. NeuroImage 17:1820-1829.

Garavan H, Ross TJ, Kaufman J, Stein EA (2003) A midline dissociation between error-processing and response-conflict monitoring. NeuroImage 20:1132-1139.

Harrison Y, Horne JA (1999) One night of sleep loss impairs innovative thinking and flexible decision making. Organ Behav Hum Decis Process 78:128-145.

Harrison Y, Horne JA (2000) The impact of sleep deprivation on decision making: a review. J Exp Psychol Appl 6:236-249.

Harrison Y, Horne JA, Rothwell A (2000) Prefrontal neuropsychological effects of sleep deprivation in young adults—a model for healthy aging? Sleep 23:1067-1073.

Hester R, Fassbender C, Garavan H (2004) Individual differences in error processing: a review and reanalysis of three event-related fMRI studies using the GO/NOGO task. Cereb Cortex 14:986-994.

Hester R, Foxe JJ, Molholm S, Shpaner M, Garavan H (2005) Neural mechanisms involved in error processing: a comparison of errors made with and without awareness. NeuroImage 27:602-608.

Horne JA (1988) Sleep loss and "divergent" thinking ability. Sleep 11:528-536.

Horne JA, Ostberg O (1976) A self-assessment questionnaire to determine morningness-eveningness in human circadian rhythms. Int J Chronobiol 4:97-110.

Jennings JR, Monk TH, van der Molen MW (2003) Sleep deprivation influences some but not all processes of supervisory attention. Psychol Sci 14:473-479.

Jones K, Harrison Y (2001) Frontal lobe function, sleep loss and fragmented sleep. Sleep Med Rev 5:463-475.

Kelly AM, Hester R, Murphy K, Javitt DC, Foxe JJ, Garavan H (2004) Prefrontal-subcortical dissociations underlying inhibitory control revealed by event-related fMRI. Eur J Neurosci 19:3105-3112.

Konishi S, Nakajima K, Uchida I, Sekihara K, Miyashita Y (1998) No-go dominant brain activity in human inferior prefrontal cortex revealed by functional magnetic resonance imaging. Eur J Neurosci 10:1209-1213.

Lawrence NS, Ross TJ, Hoffmann R, Garavan H, Stein EA (2003) Multiple neuronal networks mediate sustained attention. J Cogn Neurosci 15:1028-1038.

MacDonald III AW, Cohen JD, Stenger VA, Carter CS (2000) Dissociating the role of the dorsolateral prefrontal and anterior cingulate cortex in cognitive control. Science 288:1835-1838. 
Mazur A, Pace-Schott EF, Hobson JA (2002) The prefrontal cortex in sleep. Trends Cogn Sci 6:475-481.

Mu Q, Mishory A, Johnson KA, Nahas Z, Kozel FA, Yamanaka K, Bohning DE, George MS (2005) Decreased brain activation during a working memory task at rested baseline is associated with vulnerability to sleep deprivation. Sleep 28:433-446.

Nilsson JP, Soderstrom M, Karlsson AU, Lekander M, Akerstedt T, Lindroth NE, Axelsson J (2005) Less effective executive functioning after one night's sleep deprivation. J Sleep Res 14:1-6.

Patton JH, Stanford MS, Barratt ES (1995) Factor structure of the Barratt impulsiveness scale. J Clin Psychol 51:768-774.

Phan KL, Wager TD, Taylor SF, Liberzon I (2004) Functional neuroimaging studies of human emotions. CNS Spectr 9:258-266.

Ploghaus A, Tracey I, Gati JS, Clare S, Menon RS, Matthews PM, Rawlins JN (1999) Dissociating pain from its anticipation in the human brain. Science 284:1979-1981.

Raven J, Raven JC, Court JH (1998) Manual for Raven's advanced progressive matrices and vocabulary scales. Sec 4 , The advanced progressive matrices. Oxford: Oxford Psychologists.

Rubia K, Smith AB, Brammer MJ, Taylor E (2003) Right inferior prefrontal cortex mediates response inhibition while mesial prefrontal cortex is responsible for error detection. NeuroImage 20:351-358.

Stuss DT, Murphy KJ, Binns MA, Alexander MP (2003) Staying on the job: the frontal lobes control individual performance variability. Brain 126:2363-2380.

Sylvester CY, Wager TD, Lacey SC, Hernandez L, Nichols TE, Smith EE, Jonides J (2003) Switching attention and resolving interference: fMRI measures of executive functions. Neuropsychologia 41:357-370.

Tsai L-L, Young H-Y, Hsieh S, Lee C-S (2005) Impairment of error monitoring following sleep deprivation. Sleep 28:707-713.
Van Dongen HP (2005a) Analysis of inter- and intra-individual variability. J Sleep Res 14:201-203; author reply 205-206.

Van Dongen HP (2005b) Brain activation patterns and individual differences in working memory impairment during sleep deprivation. Sleep 28:386-388.

Van Dongen HP, Maislin G, Mullington JM, Dinges DF (2003) The cumulative cost of additional wakefulness: dose-response effects on neurobehavioral functions and sleep physiology from chronic sleep restriction and total sleep deprivation. Sleep 26:117-126.

Van Dongen HP, Baynard MD, Maislin G, Dinges DF (2004) Systematic interindividual differences in neurobehavioral impairment from sleep loss: evidence of trait-like differential vulnerability. Sleep 27:423-433.

Van Dongen HP, Vitellaro KM, Dinges DF (2005) Individual differences in adult human sleep and wakefulness: leitmotif for a research agenda. Sleep 28:479-496.

Visscher KM, Miezin FM, Kelly JE, Buckner RL, Donaldson DI, McAvoy MP, Bhalodia VM, Petersen SE (2003) Mixed blocked/event-related designs separate transient and sustained activity in fMRI. NeuroImage 19:1694-1708.

Wager TD, Sylvester CY, Lacey SC, Nee DE, Franklin M, Jonides J (2005) Common and unique components of response inhibition revealed by fMRI. NeuroImage 27:323-340.

Wang J, Rao H, Wetmore GS, Furlan PM, Korczykowski M, Dinges DF, Detre JA (2005) Perfusion functional MRI reveals cerebral blood flow pattern under psychological stress. Proc Natl Acad Sci USA 102:17804-17809.

West R, Murphy KJ, Armilio ML, Craik FI, Stuss DT (2002) Lapses of intention and performance variability reveal age-related increases in fluctuations of executive control. Brain Cogn 49:402-419.

Yamasaki H, LaBar KS, McCarthy G (2002) Dissociable prefrontal brain systems for attention and emotion. Proc Natl Acad Sci USA 99:11447-11451. 